\title{
LIQUID-FILLED SEALED MEMS CAPSULES \\ FABRICATED BY FLUIDIC SELF-ASSEMBLY
}

\author{
Massimo Mastrangeli*, Loïc Jacot-Descombes, Maurizio R. Gullo, Juergen Brugger \\ Microsystems Laboratory (LMIS1), École Polytechnique Fédérale de Lausanne (EPFL), \\ Station 2, 1015 Lausanne, Switzerland
}

\begin{abstract}
We present a new method to encapsulate functional liquids into sealed MEMS capsules by fluidic self-assembly. Self-assembly of $200 \mu \mathrm{m}$ SU-8 cargos and picoliter liquid co-encapsulation are driven by the interplay of global fluidic drag and short-range capillary forces. The latter ensues from the localized surface-selective precipitation of a photopolymerizable adhesive onto the capsules' rim. Assembly yield higher than $50 \%$ is achieved, and can be improved by optimized agitation and shape matching. The method is massively parallel, scalable and compatible with batch MEMS fabrication. It can address a variety of applications, including distributed MEMS, cell encapsulation and drug delivery.
\end{abstract}

\section{INTRODUCTION}

Liquid-filled, mechanically-rugged MEMS containers affording controlled content release are uniquely suited for important technological applications such as, e.g., targeted drug delivery [1], cell encapsulation [2] and MEMS networks [3]. Computing and sensing capabilities can be embedded in such barely visible cargos [4], whose collective potential is tackled in large distributed swarms [3]. In this perspective, optimal and cost-effective resource utilization require massive parallelism and scalability for both fabrication and assembly of microcapsules. For the former, waferscale photolithography offers unmatched yield. For the latter, dexterous servoed yet serial pick-and-place assembly of micrometric parts is hardly efficient due to stiction effects expected to worsen in presence of liquid interfaces [5]; and wafer-level bonding/sealing is challenged by liquid-related issues, as well. Conversely, the intrinsically parallel pairwise fluidic self-assembly (SA) [6] of half-capsules into full liquid-filled capsules would yield naturally to the task, if stable sealing of capsules be integrated in the process. In this respect, Whitesides' [7] and Parviz' [8] groups pioneered the fluidic SA of microcomponents mediated by the precipitation of an insoluble phase from a polar solution; and Nemani et al. evidenced the mechanical stability of SU8-based microcapsules bonded through adhesives [2].

We hereby demonstrate seamless photo-activated sealing and liquid co-encapsulation in fluidically self-assembled MEMS capsules. Our research builds on and extends the aforementioned techniques by their joint application to highthroughput encapsulation into mechanically-stable MEMS containers. This work improves our prior findings on pairwise fluidic SA mediated by hydrophobic interactions [9] by: 1) achieving significantly higher assembly selectivity, yield and throughput, and 2) enabling the mechanical sealing of the self-assembled microcapsules. As an alternative to capillary self-folding and sealing of polyhedral microcontainers [10], our SA method affords a simpler fabrication process still compatible with the embedding of functionalities (e.g. microchip [4]) within the microcapsules.

\section{MATERIALS AND METHODS}

The fluidic SA procedure and fabrication process of the microcapsules are illustrated in Figs. 1 and 2-3, respectively, and described in the following sections.

\section{Fabrication of half microcapsules}

A $100 \mathrm{~mm} \mathrm{Si}$ wafer is masked by photolithographically-patterned posts of positive tone resist (Fig. 2a) and structured by a fluorine-based, Bosch-like deep reactive ion etching (DRIE) step to obtain arrays of thousands of $50 \mu \mathrm{m}$-high triangular pillars with edgelength varying from 20 to $40 \mu \mathrm{m}$ (Fig. 2b). Cylindrical half capsules with diameter of $100 \mu \mathrm{m}$ and flat bases are then correspondingly patterned on top of the pillars out of a single, $100 \mu \mathrm{m}$-thick spun SU-8 layer (GM 1075, Gersteltec, CH; Fig. 2c). The concentric Si pillars constitute the positive for the cavities embossed in the half capsules (Fig. $3 \mathrm{I}-\mathrm{II}$ ). A $300 \mathrm{~nm}$-thick layer of $\mathrm{SiO}_{2}$ is subsequently deposited over the substrate by oxygen-rich

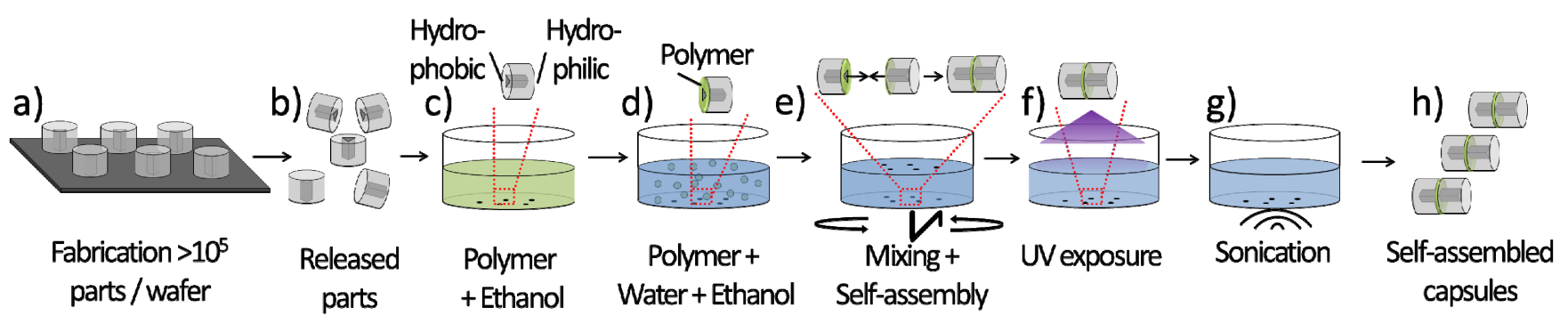

Figure 1: The fluidic process for the self-assembly and sealing of liquid-filled MEMS capsules. Self-assembly and sealing are mediated by an insoluble polymeric phase precipitated from a polar solvent. Controlled orbital agitation and sonication of the assembly solution are pivotal to the yield and throughput of the process. 
RF sputtering (Pfeiffer Spider 600) to render the exterior surface of the half capsules fully and stably hydrophilic (Figs. 2d and 3b). The half capsules are finally released from the substrate by immersion in an aqueous, heated $\mathrm{KOH}$ bath $\left(1 \mathrm{M}, 80^{\circ} \mathrm{C}\right)$ for up to 2 hours (Fig. 2e). The open (i.e. bottom) sides of the microcapsules are geometrically shielded during the $\mathrm{SiO}_{2}$ sputtering step. They preserve the rather hydrophobic character of the native SU-8 surface [9] after release. The ensuing surface energy difference between the sidewalls and the open base of the half capsules is pivotal for the selective precipitation of the polymeric phase, described in the next section.

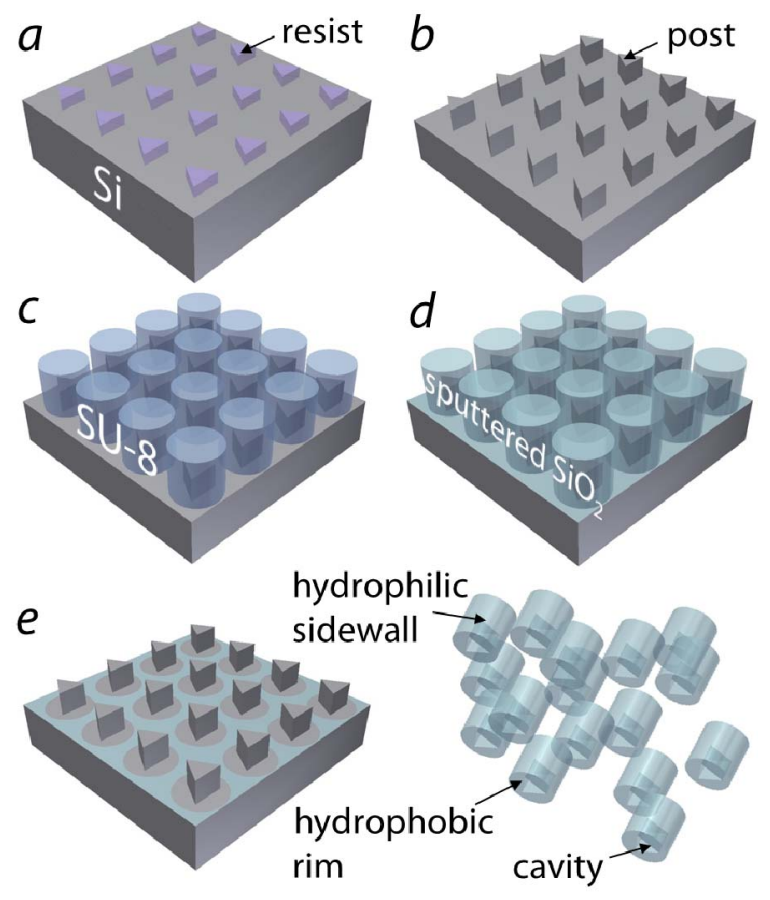

Figure 2: Fabrication of the half microcapsules. a) Lithographical resist patterning over Si substrate, b) DRIE of $50 \mu \mathrm{m}$-high triangular pillars, c) patterning of the half capsules with concentric cavities out of a single, $100 \mu \mathrm{m}$ thick SU-8 layer, d) sputtering of 300 nm-thick $\mathrm{SiO}_{2}$ over the substrate, and e) release of the half capsules in aqueous heated $\mathrm{KOH}$ bath $\left(1 \mathrm{M}, 80^{\circ} \mathrm{C}\right.$, up to 2 hours).

\section{Fluidic self-assembly procedure}

After release, the half capsules are transferred into an ethanolic solution of a photo-crosslinkable, acrylate-based polymer $(0.5 \% \mathrm{wt}$. $)$ at the bottom of a clean glass beaker (Schott-Duran, $10 \mathrm{~mL}$ ) (Fig. 1c). The solution is then diluted with 5 parts of water, and $3 \mathrm{~mL}$ are orbitally stirred (Labnet Orbit 1000) at $100 \mathrm{rpm}$ for 30' to allow for uniform mixture and selective precipitation of the polymer phase from the polar solvent onto the hydrophobic annular rim of the half capsules' open side (Fig. 1d). The consequent formation of localized thin films of hydrophobic adhesive is a result of the tailored minimization of the excess surface energy of the system [8]. Driven by the interplay of global fluidic drag and local capillary forces, the fluidic SA of full

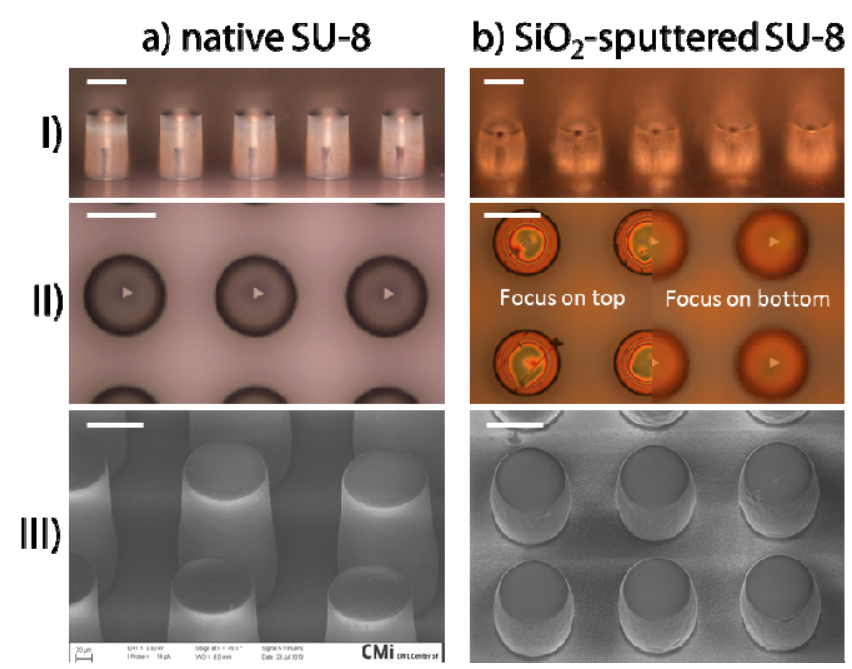

Figure 3: Optical images of the lithographical patterning (a) and $\mathrm{SiO}_{2}$ coating (b) of the $\mathrm{SU}-8$ half microcapsules. I) side views: inner Si pillars can be seen in transparency; II) top views, showing the triangular cross-section of inner pillars; III) SEM micrographs. Conformality to $S U$ 8 and surface smoothness of the sputtered $\mathrm{SiO}_{2}$ layer increase with its thickness. Scale bars are $100 \mu \mathrm{m}$.

microcapsules and concurrent liquid encapsulation take place during subsequent $30^{\prime}$ of orbital agitation at $150 \mathrm{rpm}$ (Fig. 1e). After further water dilution to remove excess polymer, the assembly solution is exposed to UV radiation for 5 ' to cross-link the interstitial adhesive films, thus enforcing the sealing of self-assembled microcapsules (Fig. 1f). The assembly solution is then sonicated by dipping the beaker in an ultrasonic water bath at $30 \mathrm{kHz}$ for 5 ' (Fig. $1 \mathrm{~g}$ ). The disposable microcapsules can finally be stored in water or in low surface tension liquids such as ethanol (Fig. 1h).

\section{RESULTS}

Photopatterning of the single SU-8 layer on top of the structured $\mathrm{Si}$ substrate results in axisymmetric half microcapsules with slightly sloped sidewalls (Fig. 3). This is attributed to 1) dispersion and pillars-induced scattering of UV light inside the thick SU-8 layer during masked exposure, and 2) the relaxation of the cylindrical structures during SU-8 development and thermal curing. Sputtering of $\mathrm{SiO}_{2}$ produces a smooth, conformal coating which does not affect the shape nor, importantly, the release of the half capsules. The detachment of the half capsules is in fact promoted by the permeation of diluted $\mathrm{KOH}$ through the SU-8/Si interfaces, in turn eased by the poor adhesion between the two materials. Half capsules with large cavities detach by slipping off the Si pillars, while small pillars remain embedded inside the half capsules after breaking off from the substrate, and are thereby dissolved by the etching bath.

Optical snapshots of a typical progression of the fluidic SA process are shown in Fig. 4. We used batches of about 500 equal half capsules per experiment. Effective low-speed orbital shaking and concurrent selective precipitation of the 

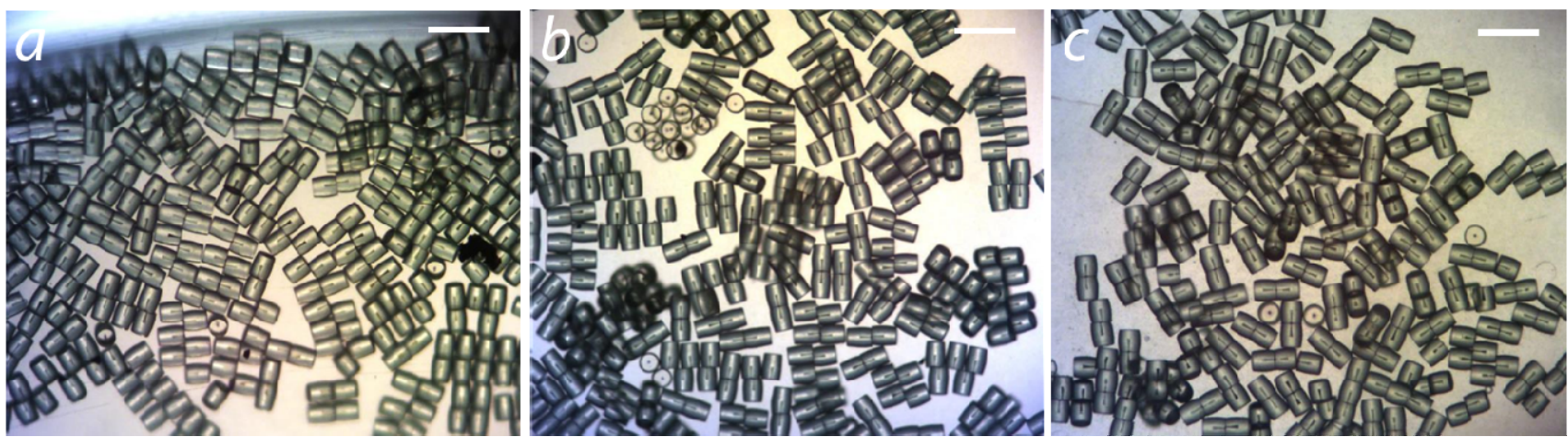

Figure 4: Optical snapshots from a typical fluidic SA run with 500 half capsules: a) after initial low-speed mixing and 10' of agitation, b) after 30' of agitation and UV exposure, c) after subsequent 5' of sonication. Scale bars are $200 \mu \mathrm{m}$.

insoluble phase from the assembly solution cause an initial, diffusion-limited aggregation of almost all the half capsules into bridging bi-layered structures (Fig. 4a). Within these extended aggregates the half capsules assume the required, face-selective orientation, yet their pair-wise alignment is mostly skewed and only partial. Upon increase of the orbital agitation speed, larger fluidic shear stresses are applied to the aggregates and can overcome the local capillary forces of the fluid menisci interposed between partially overlapping half capsule pairs. Elongated multimeric aggregates are consequently broken up, and the capillary self-alignment of facing half capsules is enhanced (Fig. 4b). After photopolymerization of the adhesive, the acousto-fluidic forces of the ultrasonic field further increase the singulation of the self-assembled full microcapsules while keeping them intact (Fig. 4c). Our open-loop fluidic SA procedure routinely achieves yield in excess of $50 \%$ in about 1 hour, as measured by the relative number of full capsules assembled out of the initial half capsules. The mechanical strength of the thin, conformal polymeric sealing is proved by 1) its resistance to ultrasonic irradiation, and 2) leakage-free encapsulation of colored ink as an instance of functional liquid (Fig. 5). Self-assembled full capsules showed no apparent degradation or leakage upon storage in water for 1 week.

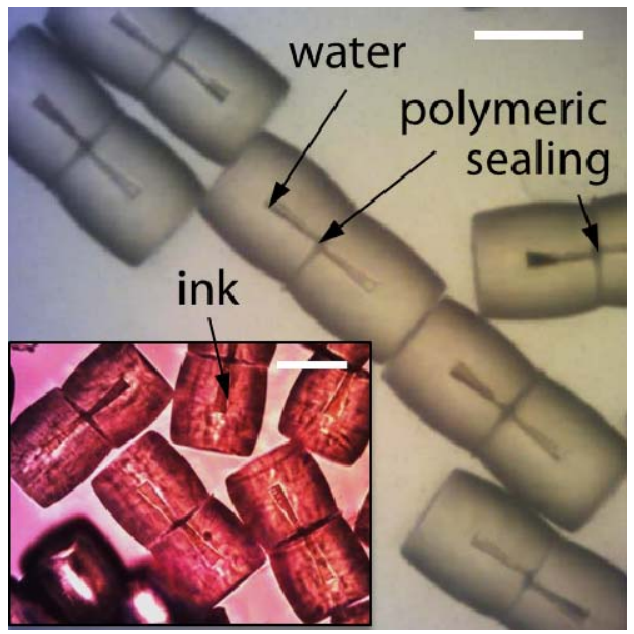

Figure 5: Sealed self-assembled full microcapsules containing water and red ink (inset). Scale bars are $100 \mu \mathrm{m}$.

\section{DISCUSSION}

Through iterative experimental optimization we elucidated three factors as key for the assembly throughput of our process: face-selective precipitation of the immiscible adhesive, control of fluidic drag forces by orbital agitation, and active use of ultrasonic energy for capsule singulation.

The spatial self-coordination of the half capsules is informed by the controlled precipitation of the immiscible adhesive onto the annular rim of their open sides. The surface selectivity is enforced by the sharp chemical contrast between the native and $\mathrm{SiO}_{2}$-coated $\mathrm{SU}-8$ surfaces of the half capsules, quantified by a water contact angle difference of $\sim 90^{\circ}$. The $\mathrm{SiO}_{2}$ coating of SU-8 preserves its full hydrophilicity upon prolonged exposure to diluted $\mathrm{KOH}$, as well. It is therefore preferable to SU-8 hydrophilization by oxygen plasma [9], besides being more stable. The energetic interface between the adhesive films and the polar solvent drives the capillary adhesion between approaching and correctly-oriented half capsules. The thickness of the precipitated film increases with time and can be controlled by the initial adhesive concentration [8]. The film thickness can easily be larger than the roughness and bowing of the SU-8 surface. Thus, related issues concerning short-range interactions [9] are avoided by the use of the liquid bridge [7].

Efficient SA is achieved by balancing the attractive forces of the localized capillary menisci with the disjoining fluidic shear stresses enacted by global orbital agitation. The magnitude of fluidic drag forces can be tuned by choosing the orbital speed in relation to: 1) the dimension of the beaker relative to the radius of orbital stirring, and 2) the volume-dependent height of the solution in the beaker [11]. Observations through an inverted microscope evidenced that, in our experimental conditions, the drag induced at 150 rpm by the laminar flow at the bottom of the beaker was effective to partially disperse large multimers - thus overcoming capillary forces of the order of $100 \mu \mathrm{N}$ [7] — while crowding the half capsules toward the center of the beaker. Conversely, half capsules motion at $100 \mathrm{rpm}$ was barely noticeable. Capsules mobility depends also on their heightto-diameter aspect ratio (AR), and is hindered by friction for $\mathrm{AR}<1$. Final, active use of ultrasonic energy proved significant to overcome residual local minima in the energy land- 


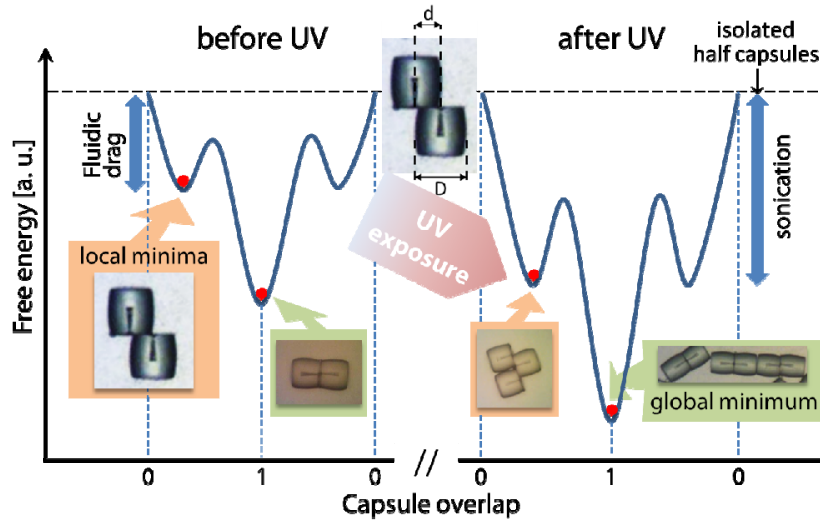

Figure 6: Sketch of the energy landscape as function of the overlap $O=1-|d| / D$ of facing half capsules pairs (see inset; D half capsules diameter, d distance between centers of open faces). Clusters of partially overlapping capsules (1.e. local energy minima) are disassembled by fluidic drag forces and ultrasonic field forces respectively before and after exposure of the solution to UV radiation, actively improving the yield of the process.

scape, i.e. to break apart remaining aggregates and increase the fraction of correct dimers after UV exposure. The consistent contributions of fluidic and ultrasonic forces to assembly yield are depicted in Fig. 6.

The process' main failure modes originate from: 1) incomplete adhesive coating of the half capsules' rims, and 2) flat geometry of the open sides of the capsules. The former hinders the complete self-alignment of capsule pairs, as they get stuck in partial overlap for lack of a continuous, hydrophobic lubricating film. This issue is eased by a longer precipitation stage prior to SA. The latter mode entails the initial formation of elongated bi-layered aggregates out of the skewed overlap of tens of half capsules. Breaking apart such aggregates, which may extend out of the horizontal plane (as seen in Fig. 4b), significantly extends the duration of the SA process. Enhanced throughput can therefore be achieved by fabricating half capsules with geometrically complementary (i.e. lock \& key) open sides, as improved shape matching can constrain exclusively dimeric capsule SA.

\section{CONCLUSIONS}

We presented an innovative fluidic SA process for the fabrication of sealed MEMS cargos encapsulating functional liquids. The process achieves an assembly yield higher than $50 \%$ for cylindrical half capsules - a figure expected to further improve through the use of more sophisticated geometries. The massively parallel nature of SA makes our fluidic encapsulation method compliant with batch MEMS fabrication and scalable to high volume manufacturing provided a consistent scaling of fluidic stirring [11]. Our biofriendly SA process is compatible with most organic liquids as it takes place in a polar solvent. It can easily be adapted to biocompatible materials other than SU-8. Particularly, the use of bioerodible polymers, such as polylactide, could allow triggerable content release and superior disposability in advanced medical and environmental applications [1].

\section{ACKNOWLEDGEMENTS}

This work was sponsored by the Nano-Tera.ch research initiative within the framework of the SelfSys project. The authors thank the great staff of EPFL's Center for Micronanotechnology for constant availability and support, Arnaud Bertsch of EPFL's LMIS4 for providing and helping with the preparation of the adhesive, and Victor J. Cadarso for insightful discussions.

\section{REFERENCES}

[1] A. P. Esser-Khan, S. A. Odom, N. R. Sottos, S. R. White, J. S. Moore, "Triggered release from polymer capsules", Macromolecules, vol. 44, pp. 5539-5553, 2011.

[2] K. Nemani, J. Kwon, K. Trivedi, W. Hu, J.-B. Lee, B. Gimi, "Biofrendly bonding processes for nanoporous implantable SU-8 microcapsules for encapsulated cell therapy", J. Microencapsul., vol. 28, pp. 771-782, 2011

[3] J. Bourgeois, S. C. Goldstein, "Distributed intelligent MEMS: Progresses and Perspectives", in ICT Innovations 2011, AISC, vol. 150, pp. 15-25, 2012

[4] L. Jacot-Descombes, M. R. Gullo, M. Mastrangeli, J. Brugger, "Inkjet printed SU-8 hemispherical microcapsules and silicon chip embedding" Micro Nano Lett., vol. 8, pp. 633-636, 2013

[5] P. Lambert, Capillary forces in microassembly, Springer, 2007

[6] N. B. Crane, O. Onen, J. Carballo, Q. Ni, R. Guldiken, "Fluidic assembly at the microscale: progress and prospects", Microfluid. Nanofluid., vol. 14, pp. 383-419, 2013

[7] A. Terfort, N. Bowden and G. M. Whitesides, "Threedimensional self-assembly of millimeter scale components", Nature, vol. 386, pp. 162-4, 1997

[8] C. J. Morris, H. Ho and B. A. Parviz, "Liquid polymer deposition on free-standing microfabricated parts for self-assembly", J. Microelectromech. Syst., vol. 15, pp. 1795-1804, 2006

[9] L. Jacot-Descombes, C. Martin-Olmos, M. R. Gullo, V. J. Cadarso, G. Mermoud, L. G. Villanueva, M. Mastrangeli, A. Martinoli, J. Brugger, "Fluid-mediated parallel self-assembly of polymeric micro-capsules for liquid encapsulation and release", Soft Matter, vol. 9, pp. 9931-9938, 2013

[10]R. Fernandes, D. H. Gracias, "Self-folding polymeric containers for encapsulation and delivery of drugs", Adv. Drug Deliver. Rev. vol. 64, pp. 1579-1589, 2012

[11] W. Weheliye, M. Yianneskis, A. Ducci, "On the Fluid Dynamics of Shaken Bioreactors-Flow Characterization and Transition", AIChE J., vol. 59, pp. 334-344, 2013

\section{CONTACT}

*Massimo Mastrangeli. Current Address: Bio, Electro And Mechanical Systems (BEAMS), École Polytechnique, Université Libre de Bruxelles, 1050 Bruxelles, Belgium. tel.: + 32265047 66, massimo.mastrangeli@ulb.ac.be 